\title{
MARKOV STATES AND CHAINS ON THE CAR ALGEBRA
}

\author{
LUIGI ACCARDI \\ Centro Interdisciplinare Vito Volterra, II Università di Roma "Tor Vergata", \\ Via Columbia 2, 00133 Roma, Italy \\ accardi@volterra.uniroma2.it \\ FRANCESCO FIDALEO \\ Dipartimento di Matematica, II Università di Roma "Tor Vergata", \\ Via della Ricerca Scientifica, 00133 Roma, Italy \\ fidaleo@mat.uniroma2.it \\ FARRUH MUKHAMEDOV \\ Department of Mechanics and Mathematics, National University of Uzbekistan, \\ Vuzgorodok, 700095, Tashkent, Uzbekistan \\ far75m@yandex.ru
}

Received 15 April 2005

Communicated by D. Petz

\begin{abstract}
We introduce the notion of Markov states and chains on the Canonical Anticommutation Relations algebra over $\mathbb{Z}$, emphasizing some remarkable differences with the infinite tensor product case. We describe the structure of the Markov states on this algebra and show that, contrarily to the infinite tensor product case, not all these states are diagonalizable. A general method to construct nontrivial quantum Markov chains on the CAR algebra is also proposed and illustrated by some pivotal examples. This analysis provides a further step for a satisfactory theory of quantum Markov processes.
\end{abstract}

Keywords: Noncommutative measure; integration and probability; quantum Markov processes; mathematical quantum statistical mechanics.

AMS Subject Classification: 46L53, 46L60, 60J99, 82B10

\section{Introduction}

Recently, Araki and Moriya ${ }^{9-12}$ have developed the statistical mechanics of systems on the CAR algebra. In particular, they have introduced the notion of "product state" on those algebras and determined their structure. On the other hand, in the case of infinite tensor products, it is known that the quantum Markov chains introduced in Refs. 1 and 2 can be realized as local perturbations of product states. It is therefore natural to investigate the possibility of constructing the CAR analogue of 
the quantum Markov states and chains. In this paper we show how to realize such an extension.

The paper is organized as follows. In Sec. 2 we propose a definition which unifies the notions of Markov state and of Markov chain extending a proposal of Ohno, ${ }^{20}$ and including all the presently known examples. In Sec. 3 we recall some known facts about the CAR algebra. Section 4 is devoted to investigate some general properties of quantum Markov states on the CAR algebra. In particular, we classify all the even Markovian conditional expectations on the CAR algebra. Such conditional expectations naturally arise in the study and the construction of quantum Markov states. In Sec. 5 we study the diagonalizable Markov states, a situation which is similar to what one finds in the case of infinite tensor product.

The main result in this paper is Sec. 6, Subsec. 6.4, where we prove that, contrarily to what happens in the tensor product case, on the Fermion algebra there exist nondiagonalizable Markov states. The structure of these states is explicitly described.

In Sec. 7 we describe a general method to construct Markov chains on the CAR algebra. Section 7 also contains concrete examples of Fermi Markov chains.

The theory of quantum Markov chains on infinite tensor products of matrix algebras (lattice systems) has been greatly enriched by the results of Fannes, Nacthergaele, Werner, ${ }^{14,15}$ Hiai, Petz, ${ }^{18}$ and Matsui ${ }^{19}$ where quantum Markov chains (more precisely, the subclass of these chains constructed in Theorem 4 of Ref. 2) are often called " $C^{*}$-finitely correlated states or algebraic states" (see also Ref. 6 for some connected results). Some of these papers also prove that quantum Markov chains emerge naturally, in several physically relevant models, as ground states of certain nearest neighbor Hamiltonians. Recently, quantum Markov states have been shown to emerge naturally also in quantum information theory. ${ }^{21}$ Our hope is that similar extensions and applications can also be found for Markov chains on the CAR algebra.

\section{Quasi Local Algebras and the Markov Propery}

By a (Umegaki) conditional expectation $E: \mathfrak{A} \mapsto \mathfrak{B} \subset \mathfrak{A}$ we mean a norm-one projection of the $C^{*}$-algebra $\mathfrak{A}$ onto a $C^{*}$-subalgebra (with the same identity $I$ ) $\mathfrak{B}$. The map $E$ is automatically a completely positive identity-preserving $\mathfrak{B}$-bimodule map. When $\mathfrak{A}$ is a matrix algebra, the structure of a conditional expectation is well known. Indeed, let $\mathfrak{A}$ be a full matrix algebra and consider the (finite) set $\left\{P_{i}\right\}$ of minimal central projections of the range $\mathfrak{B}$ of $E$, we have

$$
E(x)=\sum_{i} E\left(P_{i} x P_{i}\right) P_{i}
$$

Then $E$ is uniquely determined by its values on the reduced algebras

$$
\mathfrak{A}_{P_{i}}:=P_{i} \mathfrak{A} P_{i}=N_{i} \otimes \bar{N}_{i},
$$


where $N_{i} \sim \mathfrak{B}_{P_{i}}:=\mathfrak{B} P_{i}$ and $\bar{N}_{i} \sim \mathfrak{B}_{P_{i}}^{\prime}:=\mathfrak{B}^{\prime} P_{i} .{ }^{\text {a }}$ In fact, there exist states $\phi_{i}$ on $\bar{N}_{i}$ such that

$$
E\left(P_{i}(a \otimes \bar{a}) P_{i}\right)=\phi_{i}(\bar{a}) P_{i}(a \otimes I) P_{i} .
$$

Consider a triplet $\mathfrak{C} \subset \mathfrak{B} \subset \mathfrak{A}$ of unital $C^{*}$-algebras. A quasi-conditional expectation w.r.t. the given triplet, is a completely positive, identity-preserving linear map $E: \mathfrak{A} \mapsto \mathfrak{B}$ such that

$$
E(c a)=c E(a), \quad a \in \mathfrak{A}, \quad c \in \mathfrak{C} .
$$

Notice that, as the quasi-conditional expectation $E$ is a real map, we have

$$
E(a c)=E(a) c, \quad a \in \mathfrak{A}, \quad c \in \mathfrak{C}
$$

as well.

If $\varphi$ is a normal faithful state on the $W^{*}$-algebra $\mathfrak{A}$, the $\varphi$-conditional expectation $E^{\varphi}: \mathfrak{A} \mapsto \mathfrak{B}$ preserving the restriction of $\varphi$ to the $W^{*}$-subalgebra $\mathfrak{B}$, provides an example of quasi-conditional expectation. Namely, it is enough to choose for $\mathfrak{C}$ any unital $C^{*}$-subalgebra of $\mathfrak{B}$ contained in the $E^{\varphi}$-fixed point algebra. The $\varphi$ conditional expectation $E^{\varphi}$ is a Umegaki conditional expectation if and only if the modular group of $\varphi$ leaves globally stable the subalgebra $\mathfrak{B}$, see Ref. 3 .

Let $X$ be a fixed set, and $\mathcal{I}$ a directed family of subsets of $X$ closed under difference, equipped with a map $d: \mathcal{I} \rightarrow \mathcal{I}$ such that

(i) $\bigcup\{F \mid F \in \mathcal{I}\}=X$,

(ii) $d F \subseteq F, F \in \mathcal{I}$,

(iii) $F \subseteq G \Longrightarrow d F \subseteq d G$,

(iv) if $\left\{F_{\alpha}\right\}$ is any family in $\mathcal{I}$ such that $F_{a} \uparrow X$ (i.e. $\bigcup_{\alpha} F_{\alpha}=X$ ), then $d F_{\alpha} \uparrow X$.

This abstract formulation unifies Nelson's topological Markov property with Dobrushin's discrete $d$-Markov property.

A quasi-local algebra associated with the family $\mathcal{I}$ of subsets of $X$ is a $C^{*}$ algebra $\mathfrak{A}$ equipped with an isotonic family $\left\{\mathfrak{A}_{F}\right\}_{F \in \mathcal{I}}$ of local $C^{*}$-algebras such that $\bigcup\left\{\mathfrak{A}_{F} \mid F \in \mathcal{I}\right\}$ is dense in $\mathfrak{A}$.

Definition 2.1. For $F, G \in \mathcal{I}$ with $F \subseteq G$, a linear map $E_{G, F}: \mathfrak{A}_{G} \mapsto \mathfrak{A}_{F}$ is said to enjoy the $d$-Markov property if

$$
E_{G, F}\left(\mathfrak{A}_{G \backslash F}\right) \subseteq \mathfrak{A}_{F \backslash d F} .
$$

In the notations of Definition 2.1, consider

(i) an increasing sequence $\left\{F_{n}\right\}_{n \in \mathbb{N}}$ in $\mathcal{I}$ such that

$$
F_{n-1} \subseteq d F_{n} \subseteq F_{n} \uparrow X
$$


(ii) a sequence of $d$-Markovian quasi-conditional expectations

$$
E_{F_{n+1}, F_{n}}: \mathfrak{A}_{F_{n+1}} \mapsto \mathfrak{A}_{F_{n}}
$$

w.r.t. the triplet $\mathfrak{A}_{d F_{n}} \subseteq \mathfrak{A}_{F_{n}} \subseteq \mathfrak{A}_{F_{n+1}}$,

(iii) a state $\varphi_{F_{0}} \in \mathcal{S}\left(\mathfrak{A}_{F_{0}}\right)$.

Definition 2.2. A state $\varphi \in \mathcal{S}(\mathfrak{A})$ is called a $d$-Markov chain for the localization $\left\{\mathfrak{A}_{F_{n}}\right\}_{n \in \mathbb{N}}$, if

$$
\varphi=\lim _{n} \varphi_{F_{0}} \circ E_{F_{1}, F_{0}} \circ \cdots \circ E_{F_{n}, F_{n-1}}
$$

in the $*$-weak topology, for some sequence $\left\{E_{F_{n+1}, F_{n}}\right\}_{n \in \mathbb{N}}$ of quasi-conditional expectation as above.

The state $\varphi$ is called a $d$-Markov state for the localization $\left\{\mathfrak{A}_{F_{n}}\right\}_{n \in \mathbb{N}}$, if

$$
\varphi\left\lceil_{F_{n}} \circ E_{F_{n+1}, F_{n}}=\varphi\left\lceil_{F_{n+1}}, \quad n \in \mathbb{N}\right.\right.
$$

for some sequence $\left\{E_{F_{n+1}, F_{n}}\right\}_{n \in \mathbb{N}}$ of quasi-conditional expectation as above.

As mentioned in the introduction, this definition of $d$-Markov chain extends the notion of "generalized Markov state" introduced by Ohno (cf. Definition 2.1 of Ref. 20).

A $d$-Markov state is also a $d$-Markov chain. It is well known that the converse is not true in general.

In some interesting situation (cf. Refs. 4, 5, 7, and Proposition 4.2 below), the following statements are equivalent:

(i) $\varphi$ is a faithful $d$-Markov state,

(ii) each $E_{G, F}$ can be chosen to be the $\varphi$-conditional expectation from $\mathfrak{A}_{G}$ into $\mathfrak{A}_{F}$,

(iii) there exists a subalgebra $\mathfrak{R}_{G, F}$ with

$$
\mathfrak{A}_{d F} \subseteq \mathfrak{R}_{G, F} \subseteq \mathfrak{A}_{F} \subseteq \mathfrak{A}_{G}
$$

and a surjective Umegaki conditional expectation $E_{G, F}^{\circ}: \mathfrak{A}_{G} \mapsto \mathfrak{R}_{G, F}$ satisfying

$$
\varphi \circ E_{G, F}^{\circ}=\varphi
$$

\section{The CAR Algebra}

In this section we recall some basic notions (cf. Ref. 13) concerning the Canonical Anticommutation Relations (CAR, for short) algebra.

Let $I$ be a set. The CAR algebra over $I$ is the $C^{*}$-algebra $\mathfrak{A}$ with an identity $I_{\mathfrak{A}}$, generators $\left\{a_{j}, a_{j}^{+}\right\}_{j \in I}$, and relations

$$
\left(a_{j}\right)^{*}=a_{j}^{+}, \quad\left\{a_{j}^{+}, a_{k}\right\}=\delta_{j k} I_{\mathfrak{A}}, \quad\left\{a_{j}, a_{k}\right\}=\left\{a_{j}^{+}, a_{k}^{+}\right\}=0, \quad j, k \in I,
$$


where $\{x, y\}:=x y+y x$ denotes the anticommutator. The parity automorphism $\Theta$ of $\mathfrak{A}$, is characterized by the property

$$
\Theta\left(a_{j}\right)=-a_{j}, \quad \Theta\left(a_{j}^{+}\right)=-a_{j}^{+}, \quad j \in I,
$$

and induces on $\mathfrak{A}$ the $\mathbb{Z}_{2}$-grading $\mathfrak{A}=\mathfrak{A}_{+} \oplus \mathfrak{A}_{-}$where

$$
\mathfrak{A}_{+}:=\{a \in \mathfrak{A} \mid \Theta(a)=a\}, \quad \mathfrak{A}_{-}:=\{a \in \mathfrak{A} \mid \Theta(a)=-a\} .
$$

Elements in $\mathfrak{A}_{+}$(resp. $\left.\mathfrak{A}_{-}\right)$are called even (resp. odd).

For any subset $\Lambda \subset I, \mathfrak{A}_{\Lambda}$ denotes the $C^{*}$-subalgebra of $\mathfrak{A}$ generated by $\left\{a_{j}, a_{j}^{+} \mid j \in \Lambda\right\}$. Clearly $\Theta\left(\mathfrak{A}_{\Lambda}\right)=\mathfrak{A}_{\Lambda}$, therefore the $\mathbb{Z}_{2}$-grading is extended to each $\mathfrak{A}_{\Lambda}$, that is in obvious notations, $\mathfrak{A}_{\Lambda}=\mathfrak{A}_{\Lambda,+} \oplus \mathfrak{A}_{\Lambda,-}$. This allows to define the map $\Theta^{\Lambda}: \mathfrak{A} \mapsto \mathfrak{A}$ which is the the identity on $\mathfrak{A}_{\Lambda^{c}}$, and $\Theta$ on $\mathfrak{A}_{\Lambda}$. A state $\varphi \in \mathcal{S}(\mathfrak{A})$ is said to be even if it is $\Theta$-invariant.

If $I=\mathbb{Z}^{d}$, the space translations naturally act on $\mathfrak{A}$ as automorphisms, and it is well known that a translation invariant state $\varphi \in \mathcal{S}(\mathfrak{A})$ is automatically even, see e.g. Ref. 13, Example 5.2.21. Notice that, the CAR algebra is isomorphic to the $C^{*}$ infinite tensor product $\overline{\bigotimes_{\mathbb{Z}^{d}} \mathbb{M}_{2}(\mathbb{C})}{ }^{*}$, but the isomorphism does not preserve the natural localization. Hence, it does not intertwine with the corresponding Markov chains, see Sec. 6 .

For the convenience of the reader, we report in the case when $I$ is countable, the Klein-Wigner transformation establishing the mentioned isomorphism between the CAR algebra and the (infinite) tensor product over $I$ of $\mathbb{M}_{2}(\mathbb{C})$. Define $U_{j}:=$ $a_{j} a_{j}^{+}-a_{j}^{+} a_{j}, j=1,2, \ldots$, the last being any enumeration of $I$. Put $V_{0}:=I_{\mathfrak{A}}$, $V_{j}:=\prod_{n=1}^{j} U_{n}$,

$$
\begin{array}{ll}
e_{11}(j):=a_{j} a_{j}^{+}, & e_{12}(j):=V_{j-1} a_{j}, \\
e_{21}(j):=V_{j-1} a_{j}^{+}, & e_{22}(j):=a_{j}^{+} a_{j} .
\end{array}
$$

$\left\{e_{k l}(j) \mid k, l=1,2\right\}_{j \in I}$ provides a system of commuting $2 \times 2$ matrix-units realizing the mentioned isomorphism.

\section{Markov States on the CAR Algebra}

Let the CAR algebra $\mathfrak{A}:=\mathfrak{A}_{I}$ be on a totally ordered countable set $I$ containing, possibly a smallest element $j_{-}$or a greatest element $j_{+}$. In other words, $I$ is orderisomorphic to $\mathbb{Z}, \mathbb{Z}_{-}$or $\mathbb{Z}_{+}$and, in this identification, $j_{-}\left(j_{+}\right)$becomes equal to $-\infty$ $(+\infty) .{ }^{\mathrm{b}}$ We deal only with locally faithful even states and $\Theta$-equivariant (i.e. $\Theta E=$ $E \Theta)$ quasi-conditional expectations without further mention. This allows us to deal with examples, just the even Markov states and chains, suitable for physical applications.

The following definition specializes Definition 2.2 to the present situation.

\footnotetext{
${ }^{\mathrm{b}}$ We are omitting the simpler case $|I|<+\infty$.
} 
Definition 4.1. A state $\varphi$ on $\mathfrak{A}$ is called a Markov state if, for each $n<j_{+}$, there exists a quasi-conditional expectation $E_{n}$ w.r.t. the triplet $\mathfrak{A}_{n-1]} \subset \mathfrak{A}_{n} \subset \mathfrak{A}_{n+1}$ ] satisfying

$$
\begin{gathered}
\varphi_{n]} \circ E_{n}=\varphi_{n+1]}, \\
E_{n}\left(\mathfrak{A}_{[n, n+1]}\right) \subset \mathfrak{A}_{\{n\}} .
\end{gathered}
$$

We show that the Markov property defined above can be stated by a sequence of global quasi-conditional expectations, or equally well by sequences of local or global conditional expectations. In addition, putting $e_{n}:=E_{n}\left\lceil_{\mathfrak{A}_{[n, n+1]}}\right.$, it will be enough to consider the ergodic limits

$$
\varepsilon_{n}:=\lim _{m} \frac{1}{m} \sum_{h=0}^{m-1}\left(e_{n}\right)^{h},
$$

which give rise to a sequence of two-step conditional expectations, called transition expectations in the sequel.

Proposition 4.2. Let $\varphi$ be a state on the CAR algebra. The following assertions are equivalent.

(i) $\varphi$ is a Markov state;

(ii) the properties listed in Definition 4.1 are satisfied if we replace the quasiconditional expectations $E_{n}$ with conditional expectations $\mathcal{E}_{n}$;

(iii) for each $n<j_{+}$, there exists a conditional expectation $\mathcal{E}_{n]}: \mathfrak{A} \mapsto \mathcal{R}\left(\mathcal{E}_{n]}\right) \subset \mathfrak{A}_{n]}$ satisfying

$$
\begin{gathered}
\varphi \circ \mathcal{E}_{n]}=\varphi, \\
\mathcal{E}_{n]}\left(\mathfrak{A}_{[n}\right) \subset \mathfrak{A}_{\{n\}} ;
\end{gathered}
$$

(iv) the properties listed in (iii) are satisfied if we replace the conditional expectations $\mathcal{E}_{n]}$ with quasi-conditional expectations $E_{n]}$.

Proof. It is enough to prove (i) $\Rightarrow$ (ii) and (ii) $\Rightarrow$ (iii), the remaining implications being trivial.

(i) $\Rightarrow$ (ii) Consider the restriction $E_{n}^{k}:=E_{n}{\left\lceil\mathfrak{A}_{[k, n+1]}\right.}$ which is a completely positive, identity-preserving map leaving invariant a faithful state. Taking the ergodic limit

$$
\mathcal{E}_{n}^{k}:=\lim _{m} \frac{1}{m} \sum_{h=0}^{m-1}\left(E_{n}^{k}\right)^{h},
$$

we provide a projective sequence of conditional expectation defined on $\mathfrak{A}_{[k, n+1]}(n$ fixed) leaving invariant the state $\varphi\left[\mathfrak{A}_{[k, n+1]}\right.$.

$$
\text { The direct limit } \lim _{\substack{k j_{-}}} \mathcal{E}_{n}^{k}
$$

uniquely determines a sequence $\left\{\mathcal{E}_{n}\right\}_{n<j_{+}}$of conditional expectations fulfilling the required properties. 
(ii) $\Rightarrow$ (iii) Let $m>n$, define

$$
\mathcal{E}_{n, m}:=\mathcal{E}_{n} \circ \cdots \circ \mathcal{E}_{m-1} .
$$

As

$$
\mathcal{E}_{n, m+k}\left\lceil\mathfrak{A}_{m-1]}=\mathcal{E}_{n, m}\left\lceil\mathfrak{A}_{m-1]}, \text { the direct limit } \underset{m \overrightarrow{m \jmath}_{+}}{\lim } \mathcal{E}_{n, m}\right.\right.
$$

uniquely determines a sequence $\left\{\mathcal{E}_{n]}\right\}_{n<j_{+}}$of conditional expectations fulfilling the required properties.

Proposition 4.3. Let $\varepsilon_{n}: \mathfrak{A}_{[n, n+1]} \mapsto \mathcal{R}\left(\varepsilon_{n}\right) \subset \mathfrak{A}_{\{n\}}$ be a conditional expectation as above. The formula

$$
\mathcal{E}_{n}(x y):=x \varepsilon_{n}(y), \quad x \in \mathfrak{A}_{n-1]}, \quad y \in \mathfrak{A}_{[n, n+1]}
$$

uniquely defines a (even) conditional expectation of $\mathfrak{A}_{n+1]}$ into $\mathfrak{A}_{n]}$.

Proof. We start by noticing that, if $\varepsilon_{n}$ is even, then $\mathcal{E}_{n}$ given by (4.1) is automatically even, provided that the latter is well-defined.

Let $a \in \mathfrak{A}_{n+1}$. Then it can be written in a unique way as

$$
a=\sum c_{\left(j_{n}, j_{n+1}\right)\left(k_{n}, k_{n+1}\right)} e(n)_{j_{n} k_{n}} e(n+1)_{j_{n+1} k_{n+1}},
$$

where $c_{\left(j_{n}, j_{n+1}\right),\left(k_{n}, k_{n+1}\right)} \in \mathfrak{A}_{n-1]}$ are uniquely determined, and the products $e(n)_{j_{n} k_{n}} e(n+1)_{j_{n+1} k_{n+1}}$ given in Sec. 3, provide a system of matrix-units for $\mathfrak{A}_{[n, n+1]}$. We put for $a \in \mathfrak{A}_{n+1]}$ written as in (4.2),

$$
\mathcal{E}_{n}(a):=\sum c_{\left(j_{n}, j_{n+1}\right)\left(k_{n}, k_{n+1}\right)} \varepsilon_{n}\left(e(n)_{j_{n} k_{n}} e(n+1)_{j_{n+1} k_{n+1}}\right) .
$$

It is easy to show that $\mathcal{E}_{n}$ is a well-defined linear map. After some algebraic manipulation, one can show also that $\mathcal{E}_{n}$ is a norm-one projection onto a $*$-subalgebra of $\mathfrak{A}_{n]}$, that is a conditional expectation. We verify the complete positivity leaving the remaining details to the reader.

Fix a sequence $\left\{b_{i}\right\} \subset \mathfrak{A}_{n+1}$. We can write $b_{i}=\sum_{\alpha} c_{\alpha}^{i} e_{\alpha}$, where $\alpha$ denotes the generic index $\left(j_{n}, j_{n+1}\right)\left(k_{n}, k_{n+1}\right)$. We compute

$$
\begin{aligned}
\mathcal{E}_{n}\left(b_{i} b_{j}^{*}\right) & \equiv \mathcal{E}_{n}\left(\sum_{\alpha, \beta} c_{\alpha}^{i} e_{\alpha} e_{\beta}^{*} c_{\beta}^{j *}\right) \\
& =\mathcal{E}_{n}\left(\sum_{\alpha, \beta} c_{\alpha}^{i}\left(c_{\beta+}^{j *}+\sigma(\alpha, \beta) c_{\beta-}^{j *}\right) e_{\alpha} e_{\beta}^{*}\right) \\
& =\sum_{\alpha, \beta} c_{\alpha}^{i}\left(c_{\beta+}^{j *}+\sigma(\alpha, \beta) c_{\beta}^{j *}\right) \varepsilon_{n}\left(e_{\alpha} e_{\beta}^{*}\right) \\
& =\sum_{\alpha, \beta} c_{\alpha}^{i} \varepsilon_{n}\left(e_{\alpha} e_{\beta}^{*}\right) c_{\beta}^{j *} .
\end{aligned}
$$


Here, $\sigma(\alpha, \beta)$ is a sign depending on the parity of $e_{\alpha} e_{\beta}^{*}$ (the last being always even or odd), and the last equality follows by $\Theta$-equivariance which implies that $\varepsilon_{n}\left(e_{\alpha} e_{\beta}^{*}\right)$ has the same parity as $e_{\alpha} e_{\beta}^{*}$. As $\varepsilon_{n}$ is completely positive, the matrix $\left[\varepsilon_{n}\left(e_{\alpha} e_{\beta}^{*}\right)\right]_{\alpha, \beta}$ is positive, which implies that the matrix $\left[\mathcal{E}_{n}\left(b_{i} b_{j}^{*}\right)\right]_{i, j}$ is positive too, see e.g. Sec. IV.3 of Ref. 22.

As is stated in Proposition 4.2, the main object is the $\Theta$-equivariant transition conditional expectation $\varepsilon_{n}$. We start by investigating all the $\Theta$-invariant subalgebras of $\mathfrak{A}_{\{n\}}$. Of course, $\mathbb{C} I$ and $\mathbb{M}_{2}(\mathbb{C})$ are trivially $\Theta$-invariant. It remains open the case when the $\Theta$-invariant subalgebra is a maximal abelian subalgebra of $\mathbb{M}_{2}(\mathbb{C})$.

Proposition 4.4. We have two posibilities for the $\Theta$-invariant maximal Abelian subalgebras of the CAR algebra generated by $a, a^{+}$:

(i) one is generated by the projections $a a^{+}$and $a^{+} a$,

(ii) the other one is generated by the projections $Q_{\chi}$ and $Q_{-\chi}$, where

$$
Q_{\chi}:=\frac{1}{2}\left(I+\chi a+\bar{\chi} a^{+}\right), \quad \chi \in \mathbb{T},
$$

$\mathbb{T}$ being the unit circle.

Proof. Let $P$ be one of the minimal projection generating the algebra under consideration. Notice that $[P, \Theta(P)]=0$. Let $P=P_{+}+P_{-}$the splitting of $P$ into even and odd parts. If $\Theta(P)$ is different from $P$, then $\Theta(P)=I-P$, which is equivalent to $P=Q_{\chi}$ for some $\chi \in \mathbb{T}$. The remaining possibility is $\Theta(P)=P$, which means $P=a a^{+}$, or $P=a^{+} a$.

In the notations of Proposition 4.2, denote

$$
\varepsilon_{n}:=\mathcal{E}_{n}\left\lceil\mathfrak{A}_{[n, n+1]}, \quad n \in I \backslash\left\{j_{+}\right\} .\right.
$$

Lemma 4.5. If $\mathcal{R}\left(\varepsilon_{n}\right)=\mathfrak{A}_{\{n\}}$, then $\varepsilon_{n}\left(\mathfrak{A}_{\{n+1\},-}\right)=0$.

Proof. If $x_{n+1} \in \mathfrak{A}_{\{n+1\}}$ is odd, then $x_{n+1}$ anticommutes with $a_{n}, a_{n}^{+}$. Hence, $\varepsilon_{n}\left(x_{n+1}\right)$ anticommutes with $a_{n}, a_{n}^{+}$as well. As by $\Theta$-equivariance,

$$
\varepsilon_{n}\left(x_{n+1}\right)=\alpha a_{n}^{+}+\beta a_{n},
$$

we have

$$
a_{n} \varepsilon_{n}\left(x_{n+1}\right)=\alpha a_{n} a_{n}^{+}, \quad \varepsilon_{n}\left(x_{n+1}\right) a_{n}=\alpha a_{n}^{+} a_{n} .
$$

Using the above anticomutation properties, we infer that

$$
\alpha\left(a_{n} a_{n}^{+}+a_{n}^{+} a_{n}\right)=0,
$$

which implies $\alpha=0$. By the similar argument applied to $a_{n}^{+}$, we get $\beta=0$.

We pass to exhibit all the $\Theta$-equivariant transition expectations. By Proposition 4.3, they allow us to construct all the even Markovian conditional expectations on the CAR algebra. 
Put

$$
\begin{gathered}
P_{1}^{n}:=a_{n} a_{n}^{+}, \quad P_{2}^{n}:=a_{n}^{+} a_{n} ; \\
Q_{\chi}^{n}:=\frac{1}{2}\left(I+\chi a_{n}+\bar{\chi} a_{n}^{+}\right) .
\end{gathered}
$$

Proposition 4.6. Under the above assumptions, the following assertions hold true.

(i) If $\mathcal{R}\left(\varepsilon_{n}\right)=\mathbb{C} I$, then there exists an even state $\Phi_{n}$ on $\mathfrak{A}_{[n, n+1]}$ such that $\varepsilon_{n}(x)=$ $\Phi_{n}(x) I$.

(ii) If $\mathcal{R}\left(\varepsilon_{n}\right)=\mathfrak{A}_{\{n\},+}$, then there exist even states $\Phi_{1}^{n}, \Phi_{2}^{n}$ on $\mathfrak{A}_{\{n+1\}}$ such that, for $x \in \mathfrak{A}_{\{n\}}, y \in \mathfrak{A}_{\{n+1\}}$,

$$
\varepsilon_{n}(x y)=\operatorname{Tr}\left(x P_{1}^{n}\right) \Phi_{1}^{n}(y) P_{1}^{n}+\operatorname{Tr}\left(x P_{2}^{n}\right) \Phi_{2}^{n}(y) P_{2}^{n} .
$$

(iii) $\mathcal{R}\left(\varepsilon_{n}\right)=\mathfrak{A}_{\{n\}}$ then there exists an even state $\Psi_{n}$ on $\mathfrak{A}_{\{n+1\}}$ such that, for $x \in \mathfrak{A}_{\{n\}}, y \in \mathfrak{A}_{\{n+1\}}, \varepsilon_{n}(x y)=x \Psi_{n}(y)$.

(iv) If $\mathcal{R}\left(\varepsilon_{n}\right)$ is generated by $Q_{\chi}^{n}, Q_{-\chi}^{n}$, there exists a state $\eta_{\chi}^{n}$ on the reduced algebra $Q_{\chi}^{n} \mathfrak{A}_{[n, n+1]} Q_{\chi}^{n}$ such that

$$
\varepsilon_{n}(x)=\eta_{\chi}^{n}\left(Q_{\chi}^{n} x Q_{\chi}^{n}\right) Q_{\chi}^{n}+\eta_{\chi}^{n}\left(Q_{\chi}^{n} \Theta(x) Q_{\chi}^{n}\right) Q_{-\chi}^{n} .
$$

Proof. (i) and (ii) easily follow by (2.1), (2.2), by taking into account

$$
\mathfrak{A}_{\{n\},+} \bigvee \mathfrak{A}_{\{n+1\}} \sim \mathfrak{A}_{\{n\},+} \otimes \mathfrak{A}_{\{n+1\}},
$$

and the $\Theta$-equivariance of $\varepsilon_{n}$.

(iii) By Lemma 4.5 , if $x \in \mathfrak{A}_{\{n\}}, y \in \mathfrak{A}_{\{n+1\}}$, we have

$$
x \varepsilon_{n}(y)=x \varepsilon_{n}\left(y_{+}\right)=\varepsilon_{n}\left(x y_{+}\right)=\varepsilon_{n}\left(y_{+} x\right)=\varepsilon_{n}\left(y_{+}\right) x=\varepsilon_{n}(y) x .
$$

This means that $\varepsilon_{n}(y) \in \mathcal{Z}\left(\mathfrak{A}_{\{n\}}\right) \equiv \mathbb{C} I$. The assertion follows again by the $\Theta$-equivariance of $\varepsilon_{n}$.

(iv) It follows from (2.1), (2.2) and Proposition 4.4, by imposing the $\Theta$ equivariance of $\varepsilon_{n}$.

\section{Diagonalizable Markov States}

In this section we provide a decompostion of a class of Markov state, called diagonalizable in the sequel. These Markov states are precisely those for which case (iv) in Proposition 4.6 never appears. This allows us to give a reconstruction theorem for diagonalizable Markov states. These results parallel the analogous ones described in Ref. 4.

We leave the proof of the following to the reader.

Lemma 5.1. Let $\varphi$ be a Markov state on the CAR algebra, and $\left\{\varepsilon_{j}\right\}_{j_{-} \leq j<j_{+}}$the associated sequence of transition expectations. Then for each $k, l \in I$ with $k<l$, 
and $x_{k} \in \mathfrak{A}_{\{k\}}, \ldots, x_{l} \in \mathfrak{A}_{\{l\}}$,

$$
\begin{aligned}
\varphi\left(x_{k} \cdots x_{l}\right) & =\varphi\left(\varepsilon_{k}\left(x_{k} \varepsilon_{k+1}\left(x_{k+1} \cdots \varepsilon_{l-1}\left(x_{l-1} x_{l}\right) \cdots\right)\right)\right) \\
& =\varphi\left(\varepsilon_{k}\left(x_{k} \varepsilon_{k+1}\left(x_{k+1} \cdots \varepsilon_{l-1}\left(x_{l-1} \varepsilon_{l}\left(x_{l}\right)\right) \cdots\right)\right)\right) .
\end{aligned}
$$

Let $\Gamma \subset I \backslash\left\{j_{+}\right\}$be the set of sites $n$ such that $\mathcal{R}\left(\varepsilon_{n}\right)=\mathfrak{A}_{\{n\},+}$. Define $\mathcal{E}$ as the trace-preserving conditional expectation of $\mathfrak{A}$ onto $\overline{\mathfrak{A}_{I \backslash \Gamma} \bigvee\left(\bigvee_{n \in \Gamma} \mathfrak{A}_{\{n\},+}\right)}$

Proposition 5.2. Let $\varphi$ be a Markov state. Then $\varphi=\varphi \circ \mathcal{E}$.

Proof. Taking into account (2.1), we get if $n \in \Gamma$,

$$
\left.\varepsilon_{n}(x y)=\sum_{k=1}^{2} \varepsilon_{n}\left(P_{k}^{n} x y P_{k}^{n}\right) P_{k}^{n}\right)=\varepsilon_{n}\left(\sum_{k=1}^{2} P_{k}^{n} x P_{k}^{n} y\right)=\varepsilon_{n}(\mathcal{E}(x) y) .
$$

Hence, by Lemma 5.1 we obtain for every $k<l<j_{+}$, and $x_{k}, \ldots, x_{l}$ linear generators of $\mathfrak{A}_{[k, l]}$,

$$
\begin{aligned}
\varphi\left(x_{k} \cdots x_{l}\right) & =\varphi\left(\varepsilon_{k}\left(x_{k} \varepsilon_{k+1}\left(x_{k+1} \cdots \varepsilon_{l}\left(x_{l}\right) \cdots\right)\right)\right) \\
& =\varphi\left(\varepsilon_{k}\left(\mathcal{E}\left(x_{k}\right) \varepsilon_{k+1}\left(\mathcal{E}\left(x_{k+1}\right) \cdots \varepsilon_{l}\left(\mathcal{E}\left(x_{l}\right)\right) \cdots\right)\right)\right)=\varphi\left(\mathcal{E}\left(x_{k}\right) \cdots \mathcal{E}\left(x_{l}\right)\right)
\end{aligned}
$$

which leads to the assertion.

We divide $I \backslash\left\{j_{+}\right\}$into disjoint intervals each of which consisting of points $n$ such that $\mathcal{R}\left(\varepsilon_{n}\right)$ is trivial (i.e. $\mathbb{C} I$ or $\mathfrak{A}_{\{n\}}$ ), or $\mathcal{R}\left(\varepsilon_{n}\right)=\mathfrak{A}_{\{n\},+}$. In this way, $\Gamma=\stackrel{\circ}{\cup}_{k} \Gamma_{k}$ (where $\stackrel{\circ}{\cup}$ stands for disjoint union), and $\left.\Gamma_{k}=\right] l_{k}-1, r_{k}+1[$.

Define

$$
\Omega:=\prod_{k} \Omega_{k}, \quad \Omega_{k}:=\prod_{l_{k}-1<j<r_{k}+1}\{1,2\}, \quad \mu:=\prod_{k} \mu_{k},
$$

where $\mu_{k}$ is the Markov measure on $\Omega_{k}$ determined by the distributions $\pi_{\omega_{j}}^{j}$ at place $j$ and the transition coefficients $\pi_{\omega_{j} \omega_{j+1}}^{j}$ given by

$$
\begin{aligned}
& \pi_{\omega_{j}}^{j}=\varphi\left(P_{\omega_{j}}^{j}\right), \quad l_{k}-1<j<r_{k}+1, \quad \omega_{j}=1,2, \\
& \pi_{\omega_{j} \omega_{j+1}}^{j}=\frac{\varphi\left(P_{\omega_{j}}^{j} P_{\omega_{j+1}}^{j+1}\right)}{\varphi\left(P_{\omega_{j}}^{j}\right)}, \quad l_{k}-1<j<r_{k}, \quad \omega_{j}, \omega_{j+1}=1,2 .
\end{aligned}
$$

Notice that the range of the trace-preserving conditional expectation $\mathcal{E}$ can be described by the $C^{*}$-algebra consisting of all continuous functions $\omega \in \Omega \mapsto x(\omega) \in$ $\mathfrak{A}_{I \backslash \Gamma}$. Furthermore, the measure $\mu$ is given by the restriction of the Markov state $\varphi$ to the Abelian $C^{*}$-subalgebra generated by the projections $\left\{P_{\omega_{j}}^{j} \mid j \in \Gamma, \omega_{j}=1,2\right\}$.

Starting from the Markov state $\varphi$, consider for $\omega \in \Omega$, the product state extension (product state for short, see Ref. 11)

$$
\psi_{\omega}=\prod_{k} \psi_{k, \omega},
$$


on $\mathfrak{A}_{I \backslash \Gamma}$. Here, $\psi_{k, \omega}$ is the one-step or two-step product state on $\mathfrak{A}_{] r_{k}, l_{k+1}[\text { depending }}$ only on $\omega_{r_{k}}, \omega_{l_{k+1}}$, constructed as follows. ${ }^{\mathrm{c}}$

(i) If $l_{k+1}$ is the first element of $\Gamma$ not equal to $j_{-}$, or $\mathcal{R}\left(\varepsilon_{r_{k}+1}\right)=\mathbb{C} I$, then

$$
\psi_{k, \omega}(x):=\frac{\varphi\left(x P_{\omega_{l_{k+1}}^{l_{k+1}}}^{l_{k}}\right)}{\varphi\left(P_{\omega_{l_{k+1}}^{l_{k+1}}}^{l_{k}}\right)} ;
$$

(ii) if $r_{k}$ is the last element of $\Gamma$, or $\mathcal{R}\left(\varepsilon_{l_{k+1}-1}\right)=\mathfrak{A}_{\left\{l_{k+1}-1\right\}}$, then

$$
\psi_{k, \omega}(x):=\frac{\varphi\left(P_{\omega_{r_{k}}}^{r_{k}} x\right)}{\varphi\left(P_{\omega_{r_{k}}}^{r_{k}}\right)} ;
$$

(iii) if the interval under consideration has on the left and on the right, elements of $\Gamma$, that is it has the form $\left[r_{k}+1, l_{k+1}-1\right]$, then

$$
\psi_{k, \omega}(x):=\frac{\varphi\left(P_{\omega_{r_{k}}}^{r_{k}} x P_{\omega_{l_{k+1}}}^{l_{k+1}}\right)}{\varphi\left(P_{\omega_{r_{k}}}^{r_{k}}\right) \varphi\left(P_{\omega_{l_{k+1}}}^{l_{k+1}}\right)} ;
$$

(iv) for $r_{k}<j<l_{k+1}-1$, the two-step state $\psi_{k, \omega}(x), x \in \mathfrak{A}_{[j, j+1]}$ appears iff $\mathcal{R}\left(\varepsilon_{j}\right)=\mathbb{C} I$ and $\mathcal{R}\left(\varepsilon_{j+1}\right)=\mathfrak{A}_{\{j+1\}}$.

Notice that, by Proposition 4.6, the states $\psi_{\omega}$ are even. Finally, it is easy to show that the map

$$
\omega \in \Omega \mapsto \psi_{\omega} \in \mathcal{S}\left(\mathfrak{A}_{I \backslash \Gamma}\right)
$$

is measurable in the weak-* topology.

Theorem 5.3. Let $\varphi$ be a Markov state on the CAR algebra $\mathfrak{A}$.

Then $\varphi$ admits a direct-integral decomposition

$$
\varphi=\int_{\Omega}^{\oplus} \psi_{\omega}(\mathcal{E}(\cdot)(\omega)) \mu(d \omega),
$$

where the measure space $(\Omega, \mu)$ is defined in (5.1), (5.2), $\mathcal{E}$ is the trace-preserving conditional expectation onto $\overline{\mathfrak{A}_{I \backslash \Gamma} \bigvee\left(\bigvee_{n \in \Gamma} \mathfrak{A}_{\{n\},+}\right)}$, the state $\psi_{\omega}$ is given in (5.3) through (i)-(iv) above, and finally the integral (5.5) is understood as a $L^{1}$-direct integral (cf. Sec. IV.8 of Ref. 22).

Proof. The proof proceedes as that of Theorem 3.2 of Ref. 4, by taking into account the previous computations relative to the states $\psi_{\omega}$. We leave the details to the reader.

We pass to a reconstruction theorem which parallels the analogous one in Ref. 4.

${ }^{\mathrm{c}}$ If $l_{k+1}$ is the first element of $\Gamma$ not equal to $j_{-}$, then $r_{k}=j_{-}$. If $r_{k}$ is the last element of $\Gamma$, then $l_{k+1}=j_{+}$. We are using also intervals without the boundary elements (denoted as $] \cdot, \cdot[$ ) in order to take into account the possibilities $j_{-}=-\infty$ and/or $j_{+}=+\infty$. 
We start by choosing a subset $\Gamma \subset I \backslash\left\{j_{+}\right\}$together with a classical Markov process on $\Omega$ given in (5.1) with the Markov measure $\mu_{k}$ on $\Omega_{k}$ determined by the distributions $\pi_{\omega_{j}}^{j}>0$ at place $j$ and the transition matrices $\pi_{\omega_{j} \omega_{j+1}}^{j}>0$. For each $\omega$, form, according to the prescription (iv) above, an even one-step or two-step product state $\psi_{\omega}$ on $\mathfrak{A}_{I \backslash \Gamma}$ depending only on the boundaries $\omega_{r_{k}}, \omega_{l_{k+1}}$, of the decomposition of $\Gamma$ into connected intervals (as before, the subscript $k$ describes such a decomposition). Such states are well-defined, by taking into account Theorem 1 of Ref. 11. Moreover, the map given in (5.4) is measurable in the weak-* topology.

Define $\psi \in \mathcal{S}(\mathfrak{A})$ as

$$
\psi:=\int_{\Omega}^{\oplus} \psi_{\omega}(\mathcal{E}(\cdot)(\omega)) \mu(d \omega) .
$$

Consider, for each $n \in I \backslash\left\{j_{+}\right\}$the $\Theta$-equivariant conditional expectation

$$
\mathcal{E}_{n}: \mathfrak{A}_{n+1]} \mapsto \mathcal{R}\left(\mathcal{E}_{n}\right) \subset \mathfrak{A}_{n]}
$$

uniquely determined by setting for $x \in \mathfrak{A}_{n-1]}, x_{n} \in \mathfrak{A}_{\{n\}}, x_{n+1} \in \mathfrak{A}_{\{n+1\}}$,

(a) $\mathcal{E}_{n}\left(x x_{n} x_{n+1}\right):=x \psi\left(x_{n} x_{n+1}\right)$ if the two-step state $\psi\left(x_{n} x_{n+1}\right)$ appears in the decomposition of $\psi$, or $n=l_{k}-1$ ( $l_{k}$ being the left boundary of some interval of $\Gamma)$ and $\psi_{\omega}$ depends on $\omega_{l_{k}}$;

(b) $\mathcal{E}_{n}\left(x x_{n} x_{n+1}\right):=x x_{n} \psi\left(x_{n+1}\right)$ if the one-step state $\psi\left(x_{n+1}\right)$ appears in the decomposition of $\psi$, or $n=r_{k}+1\left(r_{k}\right.$ being the right boundary of some interval of $\Gamma$ ) and $\psi_{\omega}$ depends on $\omega_{r_{k}}$;

(c) $\mathcal{E}_{n}\left(x x_{n} x_{n+1}\right):=x \sum_{\omega_{n}=1}^{2} \operatorname{Tr}_{\mathfrak{A}_{\{n\}}}\left(x_{n} P_{\omega_{n}}^{n}\right) \frac{\psi\left(P_{\omega_{n}}^{n} x_{n+1}\right)}{\psi\left(P_{\omega_{n}}^{n}\right)} P_{\omega_{n}}^{n}$ if $n \in \Gamma$.

Theorem 5.4. Let $\psi \in \mathcal{S}(\mathfrak{A})$ in (5.6) be constructed by the prescriptions listed above. Then it is a Markov state w.r.t. the sequences $\left\{\mathcal{E}_{n}\right\}_{j_{-} \leq n<j_{+}}$of the abovementioned conditional expectations.

Proof. A straightforward computation, by taking into account all the various possibilities, see the proof of Theorem 4.1 of Ref. 4 .

We pass to descript the natural connections between the Markov property and the KMS conditions for states on CAR algebra. This provides natural applications to quantum statistical mechanics, see Refs. 4, 5, 7-10, for other analogous connections.

Suppose we have a locally faithful state on the CAR algebra $\mathfrak{A}$, then a potential $h_{\Lambda}$ is canonically defined for each finite subset $\Lambda$ of the index set $I$ by

$$
\varphi_{\left\lceil\mathfrak{A}_{\Lambda}\right.}=\operatorname{Tr}_{\mathfrak{A}_{\Lambda}}\left(e^{-h_{\Lambda}} \cdot\right) .
$$

Such a set of potentials $\left\{h_{\Lambda}\right\}_{\Lambda \subset I}$ satisfies normalization conditions

$$
\operatorname{Tr}_{\mathfrak{A}_{\Lambda}}\left(e^{-h_{\Lambda}}\right)=1,
$$


together with compatibility conditions

$$
\left(\operatorname{Tr}_{\mathfrak{B}_{\Lambda}} \otimes \operatorname{id}_{\mathfrak{A}_{\Lambda}}\right)\left(e^{-h_{\hat{\Lambda}}}\right)=e^{-h_{\Lambda}}
$$

for finite subsets $\Lambda \subset \hat{\Lambda}$, whenever $\mathfrak{A}_{\hat{\Lambda}} \cong \mathfrak{B}_{\Lambda} \otimes \mathfrak{A}_{\Lambda}$.

As the structure of diagonalizable Markov states is fully understood, the set of potentials $\left\{h_{[k, l]}\right\}$ related to $\varphi$ by (5.7) can be explicitly written, and satisfies some nice properties. Namely, it is easy to see that the above-mentioned potentials associated to a (locally faithful) Markov state have the form, for each $k \leq l$,

$$
h_{[k, l]}=H_{k}+\sum_{j=k}^{l-1} H_{j, j+1}+\hat{H}_{l} .
$$

Here, $\left\{H_{j}\right\}_{j_{-} \leq j \leq j_{+}},\left\{\hat{H}_{j}\right\}_{j_{-} \leq j \leq j_{+}},\left\{H_{j, j+1}\right\}_{j_{-} \leq j<j_{+}}$are sequences of even selfadjoint operators localized in $\mathfrak{A}_{\{j\}}, \mathfrak{A}_{[j, j+1]}$, respectively, satisfying the commutation relations

$$
\begin{array}{rlrl}
{\left[H_{j}, H_{j, j+1}\right]} & =0, & {\left[H_{j, j+1}, \hat{H}_{j+1}\right]} & =0, \\
{\left[H_{j}, \hat{H}_{j}\right]} & =0, \quad\left[H_{j, j+1}, H_{j+1, j+2}\right]=0 .
\end{array}
$$

It is then matter of routine to show that the pointwise norm-limit

$$
\lim _{\substack{k \rightarrow j_{-} \\ l \rightarrow j_{+}}} e^{-i t h_{[k, l]}} a e^{i t h_{[k, l]}}
$$

exists and defines a one-parameter automorphisms group $t \mapsto \sigma_{t}$ on the CAR algebra $\mathfrak{A}$ which admits $\varphi$ as a KMS state. Furthermore, $\varphi$ has a normal faithful extension on the von Neumann algebra $\pi_{\varphi}(\mathfrak{A})^{\prime \prime}$. In addition, as the CAR algebra is simple, $\varphi$ is automatically faithful.

\section{Some Illustrative Examples of Markov States}

In Sec. 4 we have show that there are four possibilities for the range of the transition expectations $\varepsilon_{n}$. Among them, two cases give rise to the same situation of product state. In this section, we describe, for each of these situations, the stationary Markov states, or two-step stationary, in the case of two-block factors. The most interesting case is described in Subsec. 4. It proves that, contrarily to what happens in the infinite tensor product case, not all Fermi Markov states are diagonalizable in the sense firstly described in Ref. 17 , see also Ref. 16 . We denote by $\alpha$ the one-step right shift on $\mathbb{Z}$, and set $\mathfrak{A}:=\mathfrak{A}_{\mathbb{Z}}$.

\subsection{Case $1 \mathcal{R}\left(\varepsilon_{n}\right)=\mathbb{C I}$ or $\mathcal{R}\left(\varepsilon_{n}\right)=\mathfrak{A}_{\{n\}}$}

We start by considering the case when the range of the transition expectations $\varepsilon_{n}$, $n \in \mathbb{Z}$, are always equal to $\mathbb{C} I$ or $\mathfrak{A}_{\{n\}}$. It is immediate to show (by Theorem 5.3 or by direct computation) that the Markov state $\varphi$ is the one-step product state given on the generators of $\mathfrak{A}$, by

$$
\varphi\left(x_{k} \cdots x_{l}\right)=\varphi\left\lceil\mathfrak { A } _ { \{ k \} } ( x _ { k } ) \cdots \varphi \left\lceil_{\mathfrak{A}_{\{l\}}}\left(x_{l}\right) .\right.\right.
$$


In this situation, $\varphi$ is translation-invariant iff $\varphi \Gamma_{\mathfrak{A}_{\{n\}}}=\varphi \Gamma_{\mathfrak{A}_{\{n+1\}}} \circ \alpha$.

\subsection{Case $2 \mathcal{R}\left(\varepsilon_{n}\right)=\mathfrak{A}_{\{n\},+}$}

Consider the case when the range of the transition expectations $\varepsilon_{n}$ are all equal to $\mathfrak{A}_{\{n\},+}$. Then, $\Gamma=\mathbb{Z}$ for the set $\Gamma$ introduced in the previous section, and $\mathcal{E}$ is the trace-preserving conditional expectation onto the maximal Abelian subalgebra $\mathfrak{D} \sim C(\Omega)$ generated by $P_{1}^{n} \equiv a_{n} a_{n}^{+}$and $P_{2}^{n} \equiv a_{n}^{+} a_{n}, n \in \mathbb{Z}$. As explained in Sec. 5, if $x \in \mathfrak{A}, \mathcal{E}(x)$ is represented by a continuous complex-valued function on $\Omega$. Hence, we obtain

$$
\varphi(x)=\int_{\Omega} \mathcal{E}(x)(\omega) \mu(d \omega) .
$$

Notice that, in this situation, the Markov state under consideration is the diagonal lifting to all of $\mathfrak{A}$, of the classical Markov process on $\mathfrak{D}$ obtained by $\varphi\lceil\mathfrak{D}$.

The Markov state is translation invariant iff the underlying Markov measure $\mu$ on $\Omega \equiv \prod_{\mathbb{Z}}\{1,2\}$ is translation invariant, that is iff the transition coefficients $\frac{\varphi\left(P_{k}^{j} P_{l}^{j+1}\right)}{\varphi\left(P_{k}^{j}\right)}=: \pi_{\omega_{j} \omega_{j+1}}^{j}>0$ (as $\varphi$ is supposed to be locally faithful) do not depend on $j \in \mathbb{Z}$, and all the coefficients $\varphi\left(P_{\omega_{j}}^{j}\right)=: \pi_{\omega_{j}}$ at places $j$ coincide with the unique stationary distribution for the primitive matrix $\pi:=\left[\pi_{\omega_{j} \omega_{j+1}}\right]$. Such a Markov state is the natural generalization of the Ising model to the CAR algebra.

The Hamiltonian for this Ising-like example is easily written taking into account that it is a diagonal lifting of a classical Markov chain. We report it for the sake of completeness.

$$
\begin{aligned}
H_{j} & =-\sum_{\omega_{j}}\left(\ln \pi_{\omega_{j}}^{j}\right) P_{\omega_{j}}^{j}, \quad \hat{H}_{j}=0, \\
H_{j, j+1} & =-\sum_{\omega_{j}, \omega_{j+1}}\left(\ln \pi_{\omega_{j}, \omega_{j+1}}^{j}\right) P_{\omega_{j}}^{j} P_{\omega_{j+1}}^{j+1} .
\end{aligned}
$$

Proposition 6.1. The locally faithful translation invariant Markov state $\varphi$ in the situation when $\mathcal{R}\left(\varepsilon_{n}\right)=\mathfrak{A}_{\{n\},+}, n \in I$ is exponentially mixing w.r.t. the spatial translations. Moreover, $\pi_{\varphi}(\mathfrak{A})^{\prime \prime}$ is a type $\mathrm{III}_{\lambda}$ von Neumann factor for some $\lambda \in$ $(0,1]$.

Proof. Consider, for $k=1,2, \ldots$, the Klein-Wigner transformation (cf. Sec. 3) obtaining the set $\left\{\left\{e_{m n}(k)\right\}_{m, n=1}^{2} \mid k=1,2, \ldots\right\}$ of mutually commuting matrixunits. Put, for $j \in \mathbb{Z}$,

$$
k(j):= \begin{cases}2 j+1, & j \geq 0, \\ -2 j, & j<0,\end{cases}
$$

and consider the new local structure generated by the algebras

$$
\mathfrak{B}_{\{j\}}:=\operatorname{span}\left\{e_{m n}(k(j)) \mid m, n=1,2\right\} .
$$


Obviously,

(i) $\mathfrak{A}_{\{j\},+} \subset \mathfrak{B}_{\{j\}}, j \in \mathbb{Z}$,

(ii) $\left[\mathfrak{B}_{\left\{j_{1}\right\}}, \mathfrak{B}_{\left\{j_{2}\right\}}\right]=0, j_{1} \neq j_{2}, j_{1}, j_{2} \in \mathbb{Z}$,

(iii) ${\overline{\bigvee_{j \in \mathbb{Z}} \mathfrak{B}_{\{j\}}}}^{C^{*}}=\mathfrak{A}$.

Namely, $\mathfrak{A}$ with the new filtration, is isomorphic to the standard spin algebra ${\overline{\mathbb{Z}_{\mathbb{Z}} \mathbb{M}_{2}(\mathbb{C})}}^{C^{*}}$, and $\varphi$ is a diagonal lifting of a classical Markov process with two-point state-space with primitive transition matrix. This means that $\varphi$ is exponentially mixing. In addition, $\pi_{\varphi}(\mathfrak{A})^{\prime \prime}$ is a type $\mathrm{III}_{\lambda}$ factor for some $\lambda \in(0,1]$, see e.g. Sec. 5 of Ref. 16.

Remark 6.2. Notice that $\varphi$ is a Markov state also for the tensor product localization. This is due to the fact that $\varphi$ is a lifting on $\mathfrak{A}$ of a classical Markov chain on the Abelian algebra

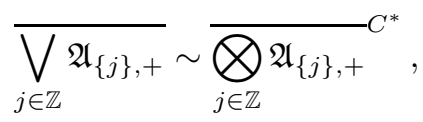

and $\mathfrak{A}_{\{j\},+} \subset \mathfrak{B}_{\{j\}}$ as well.

\subsection{Case 3 two-block factor}

Other interesting examples are the two-block factors. These (two) examples arise when the ranges of the transition expectations $\varepsilon_{n}$ are alternatively $\mathbb{C} I$ and $\mathfrak{A}_{\{\cdot\}}$, say, $\mathcal{R}\left(\varepsilon_{2 n}\right)=\mathbb{C} I$ and $\mathcal{R}\left(\varepsilon_{2 n+1}\right)=\mathfrak{A}_{\{2 n+1\}}$. In the last situation, we get

$$
\varphi\left(x_{2 k} x_{2 k+1} \cdots x_{2 l} x_{2 l+1}\right)=\varphi\left[\mathfrak { A } _ { [ 2 k , 2 k + 1 ] } ( x _ { 2 k } x _ { 2 k + 1 } ) \cdots \varphi \left[\mathfrak{A}_{[2 l, 2 l+1]}\left(x_{2 l} x_{2 l+1}\right),\right.\right.
$$

i.e. it is a two-point product state extension. It is two-step translation invariant iff $\varphi\left[\mathfrak{A}_{\mathfrak{A}_{[2 n, 2 n+1]}}=\varphi\left[\mathfrak{A}_{[2 n+2,2 n+3]} \circ \alpha^{2}\right.\right.$.

The other (isomorphic) case is obtained by shifting of one-step the previous one.

\subsection{Case $4 \mathcal{R}\left(\varepsilon_{n}\right)=\mathbb{C} Q_{\chi}^{n} \oplus \mathbb{C} Q_{-\chi}^{n}$}

We discuss the most interesting example of Fermi Markov state associated to the transition expectations satisfying the properties in (iv) of Proposition 4.6. To simplify, we restrict ourselves to the translation invariant situation.

For the $\eta_{\chi}^{n}$ in (4.4), set $\eta_{\chi}^{n}:=\eta_{\chi}^{0} \circ \alpha^{-n}$. Let $\left\{\mathcal{E}_{n}\right\}_{n \in \mathbb{N}}$ be the sequence of Umegaki conditional expectations constructed by (4.4), according to Proposition 4.2. Let

$$
\gamma:=\eta_{\chi}^{0}\left(Q_{\chi}^{0} P_{1}^{1} Q_{\chi}^{0}\right)
$$

Consider the invariant distribution for $\mathcal{E}_{0} \circ \alpha\left\lceil_{\mathfrak{A}_{\{0\}}}\right.$ whose density $w \in \mathfrak{A}_{\{0\}}$ can be easily computed as

$$
w=\gamma a_{0} a_{0}^{+}+(1-\gamma) a_{0}^{+} a_{0}
$$


With $\rho_{w}:=\operatorname{Tr}_{\mathfrak{A}_{\{0\}}}(w \cdot)$, the sequence of states

$$
\varphi_{n}:=\rho_{w} \circ \mathcal{E}_{0} \circ \cdots \circ\left(\mathcal{E}_{n}\left\lceil\mathfrak{A}_{[0, n]}\right)\right.
$$

defines a strongly clustering quantum Markov chain on $\mathfrak{A}$ (see below) which is actually a quantum Markov state as it is invariant w.r.t. the $\Theta$-equivariant transition expectation given, for $x \in \mathfrak{A}_{\{n\}}, y \in \mathfrak{A}_{\{n+1\}}$, by

$$
\tilde{\varepsilon}_{n}(x y):=\varepsilon_{n}\left(x \varepsilon_{n+1}(y)\right),
$$

with $\varepsilon_{n}$ given in (4.4).

The last Markov state is quite different from those previously considered. For example, it is expected that the local Hamiltonians $h_{[k, l]}$ defined by (5.7) does not arise from a commuting nearest neighbor interaction, and in addition, the state $\varphi$ is not diagonalizable in the language of Refs. 16 and $17 .^{\mathrm{d}}$

\section{Markov Chains on the CAR Algebra}

In this section we exhibit a general method to construct interesting classes of Markov chains on the CAR algebra.

Let $\left\{F_{n}\right\}_{n \in \mathbb{N}}$ be a collection of completely positive identity-preserving maps with $F_{n}: \mathfrak{A}_{[0, n+1]} \mapsto \mathfrak{A}_{[0, n]}$ satisfying for each $n \in \mathbb{N}$,

$$
\begin{aligned}
F_{n+1}\left\lceil\mathfrak{A}_{[0, n]}\right. & =\operatorname{id}_{\mathfrak{A}_{[0, n]},}, \\
F_{n+1} \circ \alpha\left\lceil\mathfrak{A}_{[0, n+1]}\right. & =\alpha\left\lceil_{\mathfrak{A}_{[0, n]}} \circ F_{n},\right.
\end{aligned}
$$

where $\alpha$ is the one-step right shift. Let $\rho \in \mathcal{S}\left(\mathfrak{A}_{\{0\}}\right)$ satisfy

$$
\rho=\rho \circ F_{0} \circ \alpha\left\lceil_{\mathfrak{A}_{\{0\}}} .\right.
$$

It is immediate to show that the sequence of states $\left\{\varphi_{n}\right\}_{n \in \mathbb{N}}$, where

$$
\varphi_{n}:=\rho \circ F_{0} \circ \cdots \circ\left(F_{n}\left\lceil\mathfrak{A}_{[0, n]}\right),\right.
$$

is a directed sequence. Thus, it defines a state $\varphi$ on the CAR algebra $\mathfrak{A}_{\mathbb{N}}$ which is a quantum Markov chain, according to Definition 2.2. Thanks to (7.1), (7.2), such a $\varphi \in \mathcal{S}\left(\mathfrak{A}_{\mathbb{N}}\right)$ is shift-invariant, that is it uniquely extends to a shift-invariant state (called again $\varphi$ ) on $\mathfrak{A}:=\mathfrak{A}_{\mathbb{Z}}$. We call such a shift-invariant state on $\mathfrak{A}$ the quantum Markov chain generated by the pair $\left(\left\{F_{n}\right\}_{n \in \mathbb{N}}, \rho\right)$. See also Ref. 20 for quite similar considerations.

\footnotetext{
${ }^{\mathrm{d}}$ In order to see that the last object is highly nontrivial, we compute $\varphi(x y)$, for $x \in \mathfrak{A}_{\{0\}}, y \in \mathfrak{A}_{\{1\}}$. Let $V \in \mathfrak{A}_{[0,1]}$ be the partial isometry such that $V^{*} V=Q_{-\chi}^{0}, V V^{*}=Q_{\chi}^{0}$. Put $\delta:=\eta_{\chi}^{0}\left(V\left(\chi a_{1}+\right.\right.$ $\left.\left.\left.\bar{\chi} a_{1}^{+}\right) Q_{\chi}^{0}\right)\right)$. We have in bra-ket notation,

$$
\varphi(x y)=\left\langle\chi_{0}\left|x_{+}\right| \chi_{0}\right\rangle\left\langle\chi_{1}\left|y_{+}\right| \chi_{1}\right\rangle+\delta\left\langle\chi_{0}\left|x_{-}\right| \chi_{0}^{\perp}\right\rangle\left\langle\chi_{1}\left|y_{-}\right| \chi_{1}\right\rangle
$$

where $\left|\chi_{0}\right\rangle,\left|\chi_{0}^{\perp}\right\rangle$ are the (unique up to a phase) normalized eigenvectors of $Q_{\chi}^{0}, Q_{-\chi}^{0}=Q_{\chi}^{0 \perp}$ respectively, considered as operators in $\mathfrak{A}_{\{0\}} \cong \mathbb{M}_{2}(\mathbb{C})$ (notice that $\varphi$ depends on the even part $x_{+} y_{+}+x_{-} y_{-}$of $x y$ ). Namely, nondiagonal terms involving the odd parts of $x, y$, naturally appear.
} 
For $\Lambda \subset \mathbb{Z}$, denote $\mathcal{E}_{\Lambda}^{0}$ the conditional expectation onto $\mathfrak{A}_{\Lambda}$ preserving the normalized trace $\tau$ of $\mathfrak{A}$. Let $K \in \mathfrak{A}_{[0,1],+}$ satisfy

$$
\mathcal{E}_{0]}^{0}\left(K K^{*}\right)=I \text {. }
$$

Such an operator is said to be a conditional amplitude. Define

$$
F_{n}:=\mathcal{E}_{n]}^{0}\left(\alpha^{n}(K) \cdot \alpha^{n}\left(K^{*}\right)\right)
$$

Proposition 7.1. The sequence $\left\{F_{n}\right\}_{n \in \mathbb{N}}$, with the $F_{n}$ as in $(7.4)$, defines a quantum Markov chain on the CAR algebra associated to the pair $\left(\left\{F_{n}\right\}_{n \in \mathbb{N}}, \rho\right)$, provided that $\rho \in \mathcal{S}\left(\mathfrak{A}_{\{0\}}\right)$ fulfils $(7.2)$.

Proof. Immediate by (7.3), and the fact that $K$ is even.

We pass to exhibit some pivotal examples of Markov chains on the CAR algebra. Define

$$
U=a_{1} a_{0}^{+}+a_{0} a_{1}^{+} .
$$

Put $V=\exp (\kappa U / 2)$, where $\kappa \in \mathbb{R}$. It is clear that each $V$ is positive and even. We have by (4.3), $U^{2}=P_{2}^{0} P_{1}^{1}+P_{1}^{0} P_{2}^{1}$. This means that $U^{2 n}=U^{2}, U^{2 n+1}=U$. After considering the power series defining $\exp (\kappa U)$, a simple computation leads to

$$
\exp (\kappa U)=I+(\sinh \kappa) U+(\cosh \kappa-1) U^{2} .
$$

In order to check (7.3), we use Theorem 4.7 of Ref. 10. We compute

$$
\begin{aligned}
\mathcal{E}_{0]}^{0}\left(V^{2}\right) & \equiv \mathcal{E}_{0]}^{0}(\exp (\kappa U)) \\
& =\operatorname{id}_{\mathfrak{A}_{\{0\}}} \otimes \tau\left\lceil\mathfrak { A } _ { \{ 1 \} , + } \left(\frac{1}{2}\left(\operatorname{id}_{\mathfrak{A}_{[0,1]}}+\Theta^{\{1\}}\left\lceil_{\mathfrak{A}_{[0,1]}}(\exp (\kappa U))\right)\right)\right.\right. \\
& =I+(\cosh \kappa-1) \operatorname{id}_{\mathfrak{A}_{\{0\}}} \otimes \tau\left\lceil\mathfrak{A}_{\{1\},+}\left(U^{2}\right)\right. \\
& =I+\frac{\cosh \kappa-1}{2}\left(P_{1}^{0}+P_{2}^{0}\right)=\frac{1+\cosh \kappa}{2} I .
\end{aligned}
$$

This means that

$$
K:=\sqrt{\frac{2}{1+\cosh \kappa}} V
$$

fulfills (7.3). Now, consider the linear map $\mathcal{E}_{0]}^{0}(K \alpha(\cdot) K)$ of $\mathfrak{A}_{\{0\}}$ into itself. Such a map has a matricial representation w.r.t. the canonical basis $\left\{a_{0}, a_{0}^{+}, a_{0} a_{0}^{+}, a_{0}^{+} a_{0}\right\}$ of $\mathfrak{A}_{\{0\}}$, which can be computed after some calculations, as

$$
A=\left(\begin{array}{cccc}
\frac{2 \sinh \kappa / 2}{1+\cosh \kappa} & 0 & 0 & 0 \\
0 & \frac{2 \sinh \kappa / 2}{1+\cosh \kappa} & 0 & 0 \\
0 & 0 & 1 / 2 & 1 / 2 \\
0 & 0 & 1 / 2 & 1 / 2
\end{array}\right) .
$$


Summarizing, we have the following:

Proposition 7.2. The pair $\left(\left\{F_{n}\right\}_{n \in \mathbb{N}}, \tau\left\lceil_{\mathfrak{A}_{\{0\}}}\right)\right.$, with the $F_{n}$ constructed by (7.4), (7.5), defines a Markov chain on the CAR algebra over the index set $\mathbb{Z}$ which is exponentially mixing.

Proof. By taking into account the previous considerations and Proposition 7.1, it is enough to compute the peripheral spectrum and the invariant distributions for the completely positive identity-preserving map $\mathcal{E}_{0]}^{0}(K \alpha(\cdot) K)$ whose matrix representation is given in (7.6). It is almost immediate to show that the peripheral spectrum of the symmetric matrix $A$ consists of 1 alone. By quite a standard argument, we infer that the chain is exponentially mixing, see e.g. Refs. 2 and 14. Moreover, in order to determine the unique invariant distribution, it is enough to compute the left eigenspace corresponding to the eigenvalue 1 of the matrix $\Sigma A \Sigma^{-1}$, where

$$
\Sigma:=\left(\begin{array}{llll}
0 & 1 & 0 & 0 \\
1 & 0 & 0 & 0 \\
0 & 0 & 1 & 0 \\
0 & 0 & 0 & 1
\end{array}\right) .
$$

The one-dimensional left eigenspace for $\Sigma A \Sigma^{-1}$ is generated by the left eigenvector $(0,0,1 / 2,1 / 2)$, that is the unique invariant distribution is the canonical trace on $\mathfrak{A}_{\{0\}}$.

Along the same line, it is easy to see that the conditional amplitude $R:=$ $\exp i t U, t \in \mathbb{R}$ leads to a Markov chain on $\mathfrak{A}$. Namely, (7.3) is automatically satisfied by unitarity. Furthermore, the matrix representation of $\mathcal{E}_{0]}^{0}\left(R \alpha(\cdot) R^{*}\right)$ has the form

$$
B=\left(\begin{array}{cccc}
0 & 0 & 0 & 0 \\
0 & 0 & 0 & 0 \\
0 & 0 & \frac{1+\sin ^{2} t}{2} & \frac{\cos ^{2} t}{2} \\
0 & 0 & \frac{\cos ^{2} t}{2} & \frac{1+\sin ^{2} t}{2}
\end{array}\right) .
$$

This means that, provided $t \neq \pi / 2+k \pi, k \in \mathbb{Z}$, and starting with the unique stationary distribution for $\mathcal{E}_{0]}^{0}\left(R \alpha(\cdot) R^{*}\right)$ given again by the canonical normalized trace on $\mathfrak{A}_{\{0\}}$, we exhibit another class of exponentially mixing Markov chains.

e The appearance of $\Sigma$ is explained as follows. Under our convention, to each $x \in \mathbb{M}_{2}(\mathbb{C})$ we define a column-vector $X \in \mathbb{C}^{4}$, given by

$$
X:=\left(\begin{array}{l}
x_{12} \\
x_{21} \\
x_{11} \\
x_{22}
\end{array}\right) .
$$

Then $\operatorname{Tr}(x y)=X^{t} \Sigma Y$. 


\section{References}

1. L. Accardi, Noncommutative Markov chains, in Proc. of Int. School of Math. Phys., Camerino (1974), pp. 268-295.

2. L. Accardi, On noncommutative Markov property, Funct. Anal. Appl. 8 (1975) 1-8.

3. L. Accardi and C. Cecchini, Conditional expectations in von Neumann algebras and a theorem of Takesaki, J. Funct. Anal. 45 (1982) 245-273.

4. L. Accardi and F. Fidaleo, Nonhomogeneous quantum Markov states and quantum Markov fields, J. Funct. Anal. 200 (2003) 324-347.

5. L. Accardi and F. Fidaleo, Quantum Markov fields, Inf. Dim. Anal. Quantum Probab. Rel. Topics 6 (2003) 123-138.

6. L. Accardi and F. Fidaleo, Entangled Markov chains, Ann. Mat. Pura Appl. 184 (2005) 327-346.

7. L. Accardi and A. Frigerio, Markovian cocycles, Proc. R. Ir. Acad. 83 (1983) 251-263.

8. L. Accardi and V. Liebscher, Markovian KMS states for one dimensional spin chains, Inf. Dim. Anal. Quantum Probab. Rel. Topics 2 (1999), 645-661.

9. H. Araki and H. Moriya, Local thermodynamical stability of Fermion lattice systems, Lett. Math. Phys. 62 (2002) 33-45.

10. H. Araki and H. Moriya, Equilibrium statistical mechanics of Fermion lattice systems, Rev. Math. Phys. 15 (2003) 93-198.

11. H. Araki and H. Moriya, Joint extension of states of subsystems, Commun. Math. Phys. 237 (2003) 105-122.

12. H. Araki, Conditional expectations relative to a product state and the corresponding standard potentials, Commun. Math. Phys. 246 (2004) 113-132.

13. O. Bratteli and D. W. Robinson, Operator Algebras and Quantum Statistical Mechanics, Vols. I and II (Springer, 1981).

14. M. Fannes, B. Nachtergaele and R. F. Werner, Finitely correlated states of quantum spin chains, Commun. Math. Phys. 144 (1992) 443-490.

15. M. Fannes, B. Nachtergaele and R. F. Werner, Finitely correlated pure states, J. Funct. Anal. 120 (1994) 511-534.

16. F. Fidaleo and F. Mukhamedov, Diagonalizability of nonhomogeneous quantum Markov states and associated von Neumann algebras, Probab. Math. Stat. 24 (2004) 401-418.

17. Ya V. Golodets and G. N. Zholtkevich, Markovian KMS states, Theor. Math. Phys. 56 (1983) 686-690.

18. F. Hiai and D. Petz, Entropy densities for algebraic states, J. Funct. Anal. 125 (1994) 287-308.

19. T. Matsui, A characterization of pure finitely correlated states, Inf. Dim. Anal. Quantum Probab. Rel. Topics 1 (1998) 647-661.

20. H. Ohno, Extendability of generalized quantum Markov states on gauge invariant $C^{*}$-algebras, Inf. Dim. Anal. Quantum Probab. Rel. Topics 8 (2005) 141-152.

21. D. Petz, Monotonicity of quantum relative entropy revisited, Rev. Math. Phys. 15 (2003) 79-91.

22. M. Takesaki, Theory of Operator Algebras I (Springer, 1979). 